\title{
Carotid Artery Stenosis
}

National Cancer Institute

\section{Source}

National Cancer Institute. Carotid Artery Stenosis. NCI Thesaurus. Code C95804.

A narrowing of the carotid artery lumen. It is usually caused by the formation of an

atherosclerotic plaque. Symptoms are usually present when there is severe narrowing or

obstruction of the arterial lumen and manifest as ischemic cerebrovascular accidents. 